\title{
Internet Use, Identity Development and Social Anxiety Among Young Adults
}

\author{
Dennis Mazalin and Susan Moore \\ Swinburne University of Technology, Australia
}

\begin{abstract}
Contradictory evidence exists regarding the benefit of the Internet for social and personal wellbeing, with some studies indicating deleterious effects and others possible social enrichment. The potential for increased social isolation from 'overinvolvement' in online activities or, conversely, the Internet's possibilities for enhancing social relationships, may be particularly salient during young adulthood and adolescence because of the special importance of the peer group during this developmental phase. This study was an investigation of the relationships between the levels of identity development, Internet use and social anxiety among a sample of 161 older adolescents/young adults aged between 18 and 25 . Results indicated that, for males only, higher levels of social anxiety and less mature identity statuses were associated with more frequent Internet use, specifically time spent in chatrooms, online browsing for personal use, and games. For females (who were in this sample less socially anxious, more identity-developed, and lower users of the Internet than males), social anxiety and identity status were not significantly associated with time spent online. Discussion centred around the potential roles of Internet use in reinforcing already-existing social anxiety or, alternatively, in supporting and maintaining social contacts in those with lower levels of social deficit.
\end{abstract}

$\mathrm{T}$ he Internet has evolved into a 'social technology' that is continually challenging researchers to examine its effects on numerous facets of social life (Kraut et al., 1998). The issue that attracts the most contention appears to be whether the Internet is enhancing or harming participation in community life and social relationships. Some writers emphasise its educational utility, claiming that online interactions liberate users from traditional constraints such as time and place, which results in more frequent and better social relationships. However, it is also argued that the Internet may facilitate social isolation, limit genuine social relationships, and even lead to Internet addiction, to the ultimate detriment of individual wellbeing (Wolfradt $\&$ Doll, 2001). This study examines young adults' use of the Internet and the relationships between time spent online and developmentally relevant aspects of wellbeing and social relationships - identity status and social anxiety.

In an influential longitudinal study by Kraut et al. (1998), increases in Internet use were associated with declines in the size of a person's social circle and family communication, and increases in depression and loneliness. These effects were 
stronger for adolescents than for adults. In addition, Moody (2001) found in a study of 166 university students (mean age 19.2 years) that high levels of Internet use were associated with high levels of emotional loneliness, defined as a sense of emptiness brought on by the absence of intimate relationships. This absence in intimacy was hypothesised as being a direct result of too much time being spent online, physically removed from peer relations.

Physical proximity in adolescent peer relations is believed to be largely responsible for peer acceptance or rejection (Griffiths, 1997). Griffiths reported that the ability to process social information, regulate emotions in public situations, and display acceptable and competent social behaviours are important aspects in gaining peer acceptance; and therefore the proximity of the peer group in adolescence is also important for personal growth. Furthermore, physical proximity underscores the importance of physical appearance and attraction for adolescents, particularly in the development of romantic relationships (McKenna \& Bargh, 2000). The nuances of face-to-face interactions, such as spoken words, body language and facial expressions, contribute to the social and personal development of the late adolescent/young adult (Moody, 2001).

In comparison, computer-mediated communication relies on very few cues. Short, Williams and Christie's (1976) Social Presence Theory proposes that fewer channels of communication equate to less interpersonal contact and more impersonal communication, making it harder to build relationships (Utz, 2000). However, conflicting evidence (e.g., Parks \& Floyd, 1995) has indicated that the Internet may sometimes be responsible for highly developed online relationships, some of which lead to offline social contacts, implying that social isolation may diminish with greater Internet use. In fact, McKenna and Bargh (1998), in a study of 12 Internet newsgroups, indicated that, generally, people are interested in transferring their identities and relationships nurtured from the Internet into their offline world. Furthermore, La Rose, Eastin, and Gregg (2001) replicated Kraut et al.'s (1998) study and found evidence somewhat contradictory to the original finding of high Internet use being associated with reduced social networks. They showed that Internet communication (with acquainted people) could alleviate depression. They also reported a positive relationship between Internet use and social support.

Whether males and females similarly experience the positive or negative effects of Internet use is also a matter of interest. The Internet has traditionally been a technology dominated by male users (Wolfradt \& Doll, 2001). Studies have shown that women are less interested in computers than men and display less confidence, and men have been shown to have considerably more experience (Shashaani, 1997). There is potential therefore for the sexes to show differential patterns of relationships between Internet use and social wellbeing.

With the awareness of the contradictory research literature about the social value of the Internet, some theorists (e.g., McKenna \& Bargh, 2000) have stated that it would seem erroneous and too simplistic to postulate a simple main effect of the Internet on the average person (e.g., making the person depressed or lonely). Instead, it would be more likely that differences between individuals interact with situational variables, such as various Internet applications, to produce varying behavioural and psychological results. This implies that an individual's reasons for using the Internet (or its specific applications) may determine how that person is affected by it (McKenna \& Bargh). 
Different Internet applications are associated with certain motivations to use them, or pleasures derived from them (Wolfradt $\&$ Doll, 2001). The most frequent uses of the Internet are for interpersonal communication (e.g., e-mail exchanges) and searches for information (e.g., those related to study) (Wallace, 1999). Of particular relevance for the current study is an analysis of which Internet applications are mostly associated with lower levels of adjustment or wellbeing, including features such as social anxiety. For instance, in an investigation of Internet addiction, Young (1996) found that the applications on which 'dependent' users spent most of their time $(63 \%)$ were chat and multiuser dungeons (MUDs). Wolfradt and Doll also found that the entertainment motive of Internet use, which was most strongly predicted by the games application, was positively associated with neuroticism.

Two types of wellbeing or adjustment, both of high salience to adolescents and young adults, provide the major focus of this study of Internet use. These are identity status and social anxiety. Each will be discussed in turn.

\section{Identity Development}

Erikson viewed adolescence as a pivotal period in the formation of what he termed 'ego identity', where the transition from adolescence to adulthood involved an ongoing strengthening in the sense of self (Waterman, 1982). Emanating from negotiations with a person's social reality, Erikson viewed the main psychosocial task for an adolescent as finding an optimal balance between self-knowledge and identity confusion (Kroger, 2000). He described ego identity as serving numerous functions, such as inner coherence, sameness over time and protection against sudden life changes. Erikson also emphasised peer relations in the development of a social sense of self through a process of mutual regulation between the individual and society (Schwartz et al., 2000).

Marcia operationalised the study of ego identity by introducing the possibility of qualitatively different styles (identity statuses) of adolescents' engagement in identity formation (Kroger, 2000). He identified two dimensions in the process of identity formation: exploration, where a person actively considers alternative elements of possible identities; and commitment, where they decide to adhere to a specific set of identity beliefs, values and goals (Danielsen, Lorem, \& Kroger, 2000). The 4 identity statuses postulated by Marcia (achievement, moratorium, foreclosure and diffusion) represent varying degrees of exploration and commitment. An identityachieved individual has extensively explored and finally committed himself or herself to a career, ideology, or interpersonal style. An individual who is uncommitted due to continued negotiation of the exploration process is said to be in moratorium. An individual who has committed to certain identity elements, without having explored alternatives (usually by accepting parental values unquestioningly), is in the foreclosure status. Finally, an identity-diffused individual has not made any identity commitments and is not engaged in its exploration (Schwartz et al., 2000).

Identity development within Marcia's identity-status paradigm involves movement from less mature (diffusion, foreclosure) to more mature (moratorium, achievement) identity statuses over the adolescent age range (Akers, Jones, \& Coyl, 1998). Kroger (2000) makes reference to the fact that all longitudinal investigations of identity development have found that at least $50 \%$ of participants remain diffused or foreclosed at the end of university study. The focus on identity status in the current study of late adolescents/young adults is thus still a salient one, 
as there is potential for a sample of this age group to vary widely in their level of maturity as assessed though the notion of identity.

There is potential for Internet use to facilitate or retard psychosocial development among young people, and there are also possibilities that young people at different identity statuses will be more or less motivated to use this medium. It is certainly a vehicle for exploration of the world as might characterise the lifestyle of a young person at the moratorium stage. On the other hand, high levels of Internet use may serve to limit 'real world' exploration and interaction with others, with a corresponding slowing down of the developmental journey toward identity achievement. Again, the role of gender in this relationship is of interest, given the differential Internet use patterns of the sexes (Wolfradt \& Doll, 2001) and long-standing controversies as to whether male and female adolescents follow similar pathways to identity achievement (Waterman, 1982).

\section{Social Anxiety}

Social anxiety is characterised by an intense fear of humiliation, embarrassment and negative evaluation by others in social situations, which often causes the socially anxious individual to avoid feared situations (Kashdan \& Herbert, 2001). In some cases, this acute awareness of oneself as a social object is so pronounced that the individual avoids most forms of interpersonal contact, including everyday social situations such as meeting new people or dating (Kashdan \& Herbert). At best, these encounters are endured with intense discomfort, even though the fear may be recognised as excessive or unreasonable (Stein, 2001). This presents a particularly awkward paradox as the socially anxious individual typically craves the company of others (Beck \& Emery, 1985). The mean age of onset for social anxiety is between 15 and 16 (Walsh, 2002), and it can vary across individuals, ranging from mild normative anxiety about specific events (e.g., public speaking) to a severe clinical syndrome.

The need for group affiliation and peer acceptance is often more pronounced in adolescence than in adulthood, with success in adolescent interpersonal relationships very much determined by level of proficiency of social skills. Deficits in social skills are one of the factors commonly espoused as contributing to the maintenance of social anxiety (Kashdan \& Herbert, 2001). La Greca and Lopez (1998) found that adolescents with social anxiety reported fewer friendships and close relationships, lower perceived romantic attractiveness, and less social support and acceptance from peers. The fear and avoidance characterised by social anxiety was believed to hamper the development of competent social skills, normal socialisation experiences and the overall psychological functioning of this age group (La Greca \& Lopez). Social anxiety generally affects females slightly more than males, with adolescent females typically worrying more about how others judge their appearance and behaviour (Stein et al., 2001).

McKenna and Bargh (1999) not only found that the socially anxious were more likely than the non-socially anxious to form relationships via the Internet, but that they were more likely to have formed very close (as opposed to weak) Internet friendships and romantic relationships. Therefore, for these socially anxious individuals the Internet seemed to replace some face-to-face interactions. Walther (1997) states that it is not clear whether face-to-face interactions are 'healthier' than Internet interactions. However, the emerging literature indicates that exces- 
sive online activity could become problematic if it clashes with the opportunity to participate in real-world experiences, particularly developmentally important experiences like interacting with a peer group and initiating romantic relationships. Consequently, an interesting question to answer for the current study is whether greater levels of social anxiety among adolescents are related to more frequent Internet use and, if so, which of the Internet applications are most strongly associated with social anxiety.

\section{The Current Study}

In summary, contradictory evidence has been shown to exist regarding the benefit of the Internet for social and personal wellbeing, with some studies indicating deleterious effects on social relationships, for example, Kraut et al. (1998); and others indicating possible social enrichment, for example, Park and Floyd (1995). The potential for increased social isolation from 'overinvolvement' in online activities, or conversely, the Internet's possibilities for enhancing social relationships may be particularly salient during adolescence and young adulthood. This is because of the special importance ascribed to the peer group during this developmental phase (Griffiths, 1997), including its role in the learning of social skills, and the alleviation of social anxiety. In addition, the peer group is thought to be an important conduit for identity development, facilitating independence from parents and assisting young people as they set up their own adult relationships and life-goals. Traditionally, discussions of the role of friendship/peer groups in development have assumed face-to-face interactions, and the value of an online social group in contributing to social development during adolescence and young adulthood has not been fully examined. The present study aimed to explore the association between social anxiety and Internet use among young people, acknowledging the possibility that high levels of Internet use may either open up new social possibilities or reduce them through a limitation of the time spent on face-to-face relationships. In addition, the role of developmental maturity (through the measurement of identity status) in potentially mediating any relationship between Internet use and social anxiety was explored. Finally, the roles of different types of Internet activities were considered, exploring the possibility that certain types of activities, such as use of chat rooms, might be more strongly associated with social anxiety than other online behaviours. Each of these issues was examined separately for males and females, acknowledging the possibility of different patterns of relationships for the sexes.

\section{Method}

\section{Participants}

The 161 respondents who anonymously participated were undergraduate university students from 2 campuses of a university in Melbourne, Australia. The participants were aged between 18 and 25 years and consisted of 97 females $(M=18.90$ years, $S D=1.73)$ and 64 males $(M=19.40$ years, $S D=1.70)$. The age difference between males and females was not statistically significant. The mean age of the total sample was 19.13 years $(S D=1.71)$.

\section{Materials}

Participants completed a questionnaire with items on demographics and Internet use, as well as the Extended Objective Measure of Ego Identity Status (EOM-EIS) 
(Bennion \& Adams, 1986) and the Liebowitz Social Anxiety Scale: Self-Report version (LSAS-SR) (Liebowitz, 1987).

Identity status. The EOM-EIS is a self-report scale designed to assess identity across several salient life domains. It enables total scores to be calculated for the 4 identity statuses (achievement, moratorium, foreclosure and diffusion) across these domains. The original scale contained 64 items; however, the length of this measure was reduced to 32 items, based on an adaptation of the scale by Akers, Jones, and Coyl (1998). The adaptation involved reducing the original 8 domains to 4 (occupation, philosophical lifestyle, friendship, dating). Akers et al. found that the modified scale displayed internal reliability characteristics higher than those reported for the full EOM-EIS (achievement alpha $=.74$, moratorium alpha $=.71$, foreclosure alpha $=.79$ and diffusion alpha $=.78$ ).

Each item is rated on a 6 -point Likert scale: $1=$ strongly agree, 2 = moderately agree, 3 = agree, $4=$ disagree, $5=$ moderately disagree and $6=$ strongly disagree, with ratings summed to produce 4 identity-status subscale scores. In the current study, Cronbach alpha reliabilities for the scales were $.72, .80, .72$ and .70 for diffusion, foreclosure, moratorium and achievement respectively.

Internet use. An Internet-use measure was based on Wolfradt and Doll's (2001) Internet-motivation scale. Respondents were asked to indicate which of a list of 8 computer applications they had used (yes/no response), and to estimate the amount of time spent on those applications in a typical week, on a 4-point scale $(1=$ less than 1 hour, $2=1-5$ hours, $3=5-10$ hours and $4=$ more than 10 hours). The applications were: chatrooms, e-mail, online shopping/banking, online browsing (study/work use), online browsing (personal use), newsgroups, online games and online help/support groups. An index of the amount of time spent online was calculated by summing the responses across the 8 items to produce a scale with an alpha reliability of .65 .

Social anxiety. The LSAS-SR was designed to assess the range of fear and avoidance associated with social interaction and social performance. The scale has been employed in various clinical studies and research into social-anxiety disorder (Fresco et al., 2001; Cox et al., 1998). The measure includes 24 items, 11 concerning social situations and 13 concerning performance anxiety. Participants rate each item separately for fear $(0=$ none, $1=$ mild, $2=$ moderate, $3=$ severe $)$ and avoidance behaviour ( 0 = never, 1 = occasionally, 2 = often, 3 = usually). Cronbach alpha reliabilities for the Total Fear and Total Avoidance subscales were both .91, and .95 for the total (combined) scale.

\section{Procedure}

The Human Research Ethics Committee of the university granted permission to conduct the study. Participants were recruited from psychology and computer science/information technology courses across two campuses of the university in order to obtain a good spread of extent of Internet use. Students were approached in large lectures to volunteer and those who agreed were given the questionnaire to complete in their own time. They returned the survey anonymously, either in a mailback envelope or to specially marked boxes at the university campuses. The survey took 20 to 30 minutes to complete, and the response rate of those who volunteered (i.e., took home a questionnaire) was approximately 70 percent. 
TABLE 1

Gender Differences in Levels of Social Anxiety, Identity Status, and Internet Use

\begin{tabular}{lccccc} 
& \multicolumn{2}{c}{ Males $(n=60)$} & \multicolumn{2}{c}{ Females $(n=92)$} & \\
Variable & $M$ & $S D$ & $M$ & $S D$ & $F(7,144)$ \\
\hline Social anxiety & & & & & \\
Total fear & 28.30 & 12.80 & 22.17 & 10.21 & $10.67^{* *}$ \\
Total avoidance & 25.62 & 13.64 & 20.07 & 11.04 & $7.61^{* *}$ \\
Identity & & & & & \\
Diffusion & 27.50 & 9.01 & 23.85 & 4.86 & $10.18^{* *}$ \\
Foreclosure & 18.58 & 6.51 & 15.40 & 5.83 & $9.86^{* *}$ \\
Moratorium & 27.08 & 6.67 & 27.07 & 5.70 & .00 \\
Achieved & 26.15 & 7.00 & 29.77 & 5.61 & $12.42^{* *}$ \\
Internet use & 9.02 & 4.07 & 6.28 & 2.77 & $24.24^{* * *}$ \\
\hline
\end{tabular}

Note: ${ }^{*} p<.01 .{ }^{* *} p<.001$.

\section{Results}

\section{Gender Differences}

A multivariate analysis of variance analyses was performed to test for gender differences in social anxiety (total fear, total avoidance), identity statuses (diffusion, foreclosure, moratorium, achievement), and Internet use (Table 1 shows means, standard deviations and univariate $F$ values). The overall MANOVA was significant, $F(7,144)=6.01, p<.001$. Univariate tests revealed that males scored significantly higher on both social-anxiety subscales, the diffusion and foreclosure identity statuses, and on Internet use. Females scored significantly higher on identity achievement.

\section{Social Anxiety, Identity Status and Internet Use}

The correlations between social anxiety, identity status and Internet use are presented separately in Table 2 for the sexes (males above diagonal, females below). For males, Internet use was correlated significantly with all the psychosocial variables except moratorium status. The correlations indicated that more Internet use was associated with lower levels of wellbeing, that is, higher social anxiety and less mature identity statuses. For females, there were no relationships between Internet use and either social anxiety or identity status. The total-fear and total-avoidance subscales were strongly correlated. To avoid violating multicollinearity assumptions, the total of these two subscales (LSAS-SR Total) was used in subsequent regression analyses.

A hierarchical regression analysis was performed to examine the relative 96 strength of social anxiety and identity statuses in predicting Internet use for young men. The LSAS-SR total and chronological age were entered on the first step, for which the regression equation was significant, $F(1,58)=30.17, p<.001)$. With the possible effect of social anxiety and age controlled for, the four identity statuses were entered on the second step. The pattern of results (Table 3) showed the regression equation remained significant, but that the 4 identity statuses were nonsignificant predictors of Internet use once the contribution of social anxiety and 
TABLE 2

Correlations: Social Anxiety and Identity Status with Internet Use for Both Sexes

\begin{tabular}{lcccccccc}
\hline Subscales & T fear & T avoid & LSAS & Diff & Fore & Mor & Ach & Internet \\
\hline T fear & - & .89 & .97 & .56 & $.25^{*}$ & .40 & -.47 & .59 \\
T avoid & .88 & - & .97 & .58 & $.31^{*}$ & .35 & -.49 & .57 \\
LSAS & .97 & .97 & - & .59 & $.29^{*}$ & .39 & -.49 & .59 \\
Diff & .29 & .35 & .33 & - & .34 & .47 & -.58 & .43 \\
Fore & $.09^{\mathrm{ns}}$ & $.11^{\mathrm{ns}}$ & $.12^{\mathrm{ns}}$ & .35 & - & $.04^{\mathrm{ns}}$ & $-.06^{\mathrm{ns}}$ & .35 \\
Mor & .30 & .32 & .32 & .48 & $.22^{*}$ & - & -.37 & $.23^{\mathrm{ns}}$ \\
Ach & $-.01^{\mathrm{ns}}$ & $-.02^{\mathrm{ns}}$ & $-.02^{\mathrm{ns}}$ & $-.08^{\mathrm{ns}}$ & $-.03^{\mathrm{ns}}$ & $-.11^{\mathrm{ns}}$ & - & $-.29^{*}$ \\
Internet & $.01^{\mathrm{ns}}$ & $.06^{\mathrm{ns}}$ & $.04^{\mathrm{ns}}$ & $.07^{\mathrm{ns}}$ & $-.02^{\mathrm{ns}}$ & $.08^{\mathrm{ns}}$ & $-.02^{\mathrm{ns}}$ & - \\
\hline
\end{tabular}

Note: Correlations for males above diagonal; females below diagonal. $T$ fear $=$ total fear; $T$ avoid $=$ total avoidance; LSAS = Liebowitz Social Anxiety Scale (Self-Report) total score; Diff = diffusion; Fore = foreclosure; Mor = moratorium; Ach = achievement. All values are significant at $p<.01$ except for those marked ${ }^{*}(p<.05)$, or ${ }^{\text {ns }}$ (nonsignificant).

age were accounted for. At both stage 1 and stage 2, high social anxiety and older age were predictive of greater Internet use for young men.

To examine whether particular types of Internet use were differentially associated with the psychosocial variables, correlations between time spent on each of the 8 different Internet activities and the social anxiety and identity variables were calculated separately for the sexes. Because of the large number of correlations involved $(N=80)$, a more stringent alpha level of $p<.01$ was adopted. For females, there were no significant correlations between time spent on any of the applications and the wellbeing variables. For males, there were no significant correlations between the psychosocial variables and time spent on e-mail, shopping, study or accessing news or support groups on the Internet. Table 4 indicates that, for males, time spent in chatrooms was significantly positively associated with higher social anxiety and less mature identity status. Time spent on personal online browsing

\section{TABLE 3}

Regression Analyses: Predicting Internet Use (Males)

\begin{tabular}{lc}
\hline Variable & Beta weights \\
\hline LSAS-SR total & $0.62^{* * *}$ \\
Age & $0.31^{* *}$ \\
Diffusion & 0.21 \\
Foreclosure & 0.14 \\
Moratorium & 0.00 \\
Achievement & -0.56 \\
\hline$F$ & $22.22^{* * *}$ \\
$d f$ & 2,57 \\
$R^{2}$ & 0.44 \\
\hline
\end{tabular}

Note: LSAS-SR total $=$ Liebowitz social anxiety total.

$$
{ }^{* *} p<.01,{ }^{* *} p<.001 \text {. }
$$


TABLE 4

Correlations: Social Anxiety, Identity Statuses and Internet Applications for Males

\begin{tabular}{llllll}
\hline Internet applications & LSAS & $\mathrm{D}$ & $\mathrm{F}$ & $\mathrm{M}$ & $\mathrm{A}$ \\
\hline Chat & $.59^{*}$ & $.54^{*}$ & $.35^{*}$ & .28 & -.28 \\
Personal & .30 & .25 & $.34^{*}$ & $.39^{*}$ & -.14 \\
Games & $.42^{*}$ & .20 & .10 & -.02 & -.24 \\
\hline
\end{tabular}

Note: LSAS = Liebowitz Social Anxiety Scale (Self-Report) total score; $D$ = diffusion; $F=$ foreclosure; $\mathrm{M}=$ moratorium; $\mathrm{A}$ = achievement; Personal = personal online browsing; ${ }^{*} p<.01$.

showed a similar, though weaker, pattern of correlations. Finally, more time on Internet games was associated with higher levels of social anxiety.

Separate hierarchical regression analyses were performed to test the strength of the independent variables in predicting specific Internet uses for males (chat, personal online browsing and games). In each of the analyses the LSAS-SR total and chronological age were entered on the first step. With the possible effect for social anxiety and age controlled, the 4 identity statuses were then entered on the second step. Table 5 provides the standardised beta coefficients for the hierarchical regression analyses.

At stage 1, all regression equations were significant: chat, $F(2,58)=15.90, p<$ .001 ; personal, $F(2,58)=4.24, p<.05$; games: $F(2,58)=8.72, p<.001)$. LSASSR was a stage 1 -significant predictor for all applications, while younger age was a weakly significant predictor $(p<.05)$ of Internet use for games only. At stage 2 , Table 5 indicates that all three regressions were significant, LSAS-SR retaining its strong predictive power for Internet chat and games, but dropping out as a predictor for use of the Internet for personal online browsing. Higher levels of the identity statuses of foreclosure and moratorium predicted this latter application. Age dropped out as a predictor in stage 2. Overall, patterns of relationships for males suggested higher Internet use associated with higher social anxiety and less mature identity, particularly for certain Internet applications.

\section{TABLE 5}

Regression Analyses for Prediction of Online Applications for Males

\begin{tabular}{lccc}
\hline Variable & Chat & $\begin{array}{c}\text { Beta weights } \\
\text { Personal }\end{array}$ & Games \\
\hline LSAS-SR & $0.44^{* *}$ & 0.15 & $0.45^{* *}$ \\
Age & 0.00 & 0.10 & -0.24 \\
Diffusion & 0.30 & -0.10 & -0.04 \\
Foreclosure & 0.13 & $0.30^{*}$ & 0.04 \\
Moratorium & 0.01 & $0.38^{* *}$ & -0.21 \\
Achievement & 0.13 & 0.02 & -0.07 \\
\hline$F$ & $6.87^{* * *}$ & $3.54^{* *}$ & $3.39^{* *}$ \\
$d f$ & 4,54 & 4,54 & 4,54 \\
$R^{2}$ & 0.43 & 0.28 & 0.27 \\
\hline
\end{tabular}

Note: LSAS-SR total $=$ Liebowitz social anxiety total. ${ }^{*} p<.05 .{ }^{* *} p<.01 .{ }^{* *} p<.001$. 


\section{Discussion}

In this study, the major finding was that Internet use (particularly the use of chatrooms and personal online browsing) was associated with less mature identity status and higher social anxiety for young men, but not for young women. This result can be considered in the context of the physical proximity argument offered by Griffiths (1997), and Short et al.'s (1976) Social Presence Theory. Both these researchers emphasise the learning and regulation of social information and social nuances (e.g., body language and facial expressions) through face-to-face interaction with others, and the limitation to such processes caused by reducing the number of channels of communication available in any interaction (as occurs in virtual, as contrasted with real-life, interactions).

Diminished proximity of the peer group is therefore expected to limit social learning and, given the role attributed to the peer group in adolescent development, ultimately curtail the development of mature identity (Walther, 1997). This is not due to the Internet being inherently harmful, rather that it detracts from opportunities to form valuable offline experiences. Erikson, for example, emphasised the importance of regulation between adolescents and their peer group in the development of a sense of self (Schwartz et al., 2000). Much of Erikson's discussion of the importance of peer group implies a 'real' as opposed to a 'virtual' peer network. Marcia, too, discussed the role of the peer group in enabling exploration of roles, determining of attitudes, and shaping of behaviours, all of which were seen as precursors to identity-achievement (Danielsen, Lorem, \& Kroger, 2000). McKenna and Bargh (2000) further argued that the development of romantic relations (also an important step in the path to identity-achievement) was dependent on the physical accessibility of the peer group. Thus, high Internet use may be seen as limiting access to face-to-face peer-group interactions with a resultant reduction in opportunities for social learning and the development of identity.

Although conflicting evidence exists regarding the supposed benefits of the Internet for personal and social functioning (e.g., Kraut et al., 1998; Parks \& Floyd, 1995), the current result for males was compatible with Moody's (2001) findings of increased emotional loneliness being associated with more time spent on the Internet, and Kraut et al.'s (1998) work on reduced social networks (and lowered mood) being associated with higher levels of Internet use.

Only some Internet applications related to lower wellbeing for young men. Time spent on e-mail or studying via the Internet was not related to social anxiety or weaker identity, for example. However, the use of the chatrooms and personal online browsing sites by young men was associated with less-mature identity, consistent with the McKenna and Bargh (1998) analysis that these applications are often an attraction for the 'identity poor', who use them to experiment with new roles and ways of being. Social anxiety for young men was also associated with the chat, games and personal online browsing Internet applications. This was compatible with the findings of Young (1996), whose research into Internet addiction indicated that the application most used by 'dependent' Internet-users was the chat application. Due to the powerful impact of social-avoidance behaviours reported by Kashdan and Herbert (2001), chatrooms may offer an enticing online alternative to real-life interactions for shy and socially-anxious young men. The current result was also consistent with the findings of Wolfradt and Doll (2001), who found in 
their study that the games application related to lower levels of adjustment, measured by neuroticism.

For young women, the current results did not support an association between high Internet use and less formation of mature identity or elevated social anxiety. Indeed, Internet use for young women was independent of wellbeing as assessed in this study. Why were the patterns of results different between the sexes? One possible explanation relates to the lower social anxiety, more-mature identity status and lower levels of Internet use of this sample of young women compared with the young men. These young women were, despite their similar age to the young men, generally showing higher rates of social development. Although contemporary trends indicate few gender differences in identity development (Akers, Jones, \& Coyl, 1998), the more mature (achieved) identity development of females in the current study may suggest that they have nurtured more extensive social networks and face-to-face peer relations than males. Possibly these females did not require as much use of the Internet to maintain satisfying levels of social interaction, and did not need to use the Internet to avoid face-to-face (and potentially anxiety-provoking) social relationships.

Other research has found contradictory evidence in relation to the effects of the Internet on adolescents, which, for the current female sample, may provide an insight into the contrasting results obtained between the sexes. For instance, Parks and Floyd (1995) reported that some Internet relationships can become highly developed and may lead to less social isolation; La Rose, Eastin, and Gregg (2001) found that Internet use can alleviate depression; and McKenna and Bargh (1998) found that the Internet can enhance a sense of identity and act as a catalyst for offline relationships. Young women in this study may have used their Internet time partly to support, secure and develop their offline relationships, rather than substitute for them.

The current study had several limitations which could be addressed in further research. Due to the cross-sectional methodology, statements about causal relationships could not be made (Bradley \& Matsukis, 2000). For instance, it was not possible to determine whether social anxiety contributed to more frequent Internet use or vice versa. Future studies could consider the use of longitudinal methodologies in order to further illuminate developmental pathways between social anxiety, identity development and Internet use. Nevertheless, it is conceivable that feedback cycles maintain social anxiety in late adolescents/young adults through the following causal pathways. Avoidance behaviours induce deficits in social skills, which in turn lead to delayed maturing of identity and further increases in social anxiety. Social anxiety can be maintained through avoidance behaviours, because reducing social interaction by increased Internet use can lead to a lowering of anxiety and subsequent reinforcement of the avoiding behaviour (i.e., through operant-conditioning processes). A cycle is set up of greater Internet use and reduced face-to-face contact. Such a cycle is less likely to take hold among young people whose levels of social anxiety were lower in the first place because the reinforcement of avoiding the stresses and strains of real-life relationships will be weaker.

Other limitations relate to the relatively small number of males in the sample, the nonavailability of a clinical sample with respect to social anxiety which could be compared with the normal samples, and the use of a student-only sample base. In addition, time spent online was assessed using categories (e.g., 5-10 hours) in order to assist participants in the approximation of responses. Observational meth- 
ods such as computer-generated assessments of log-on times would be more accurate than self-report measures of Internet use.

In summary, the results of the present study indicated that young men (but not young women) with less mature identities and greater levels of social anxiety tended to use the Internet more frequently for specific entertainment-related activities including chatrooms, online personal browsing and games. In order to shed further light on causal pathways, future investigations should consider using longitudinal methods, especially if they incorporate significant stages of social transitions such as the beginning of university or work.

\section{References}

Akers, J.F., Jones, R.M., \& Coyl, D.D. (1998). Adolescent friendship pairs: Similarities in identity status development, behaviours, attitudes, and intentions. Journal of Adolescent Research, 13, 178-202.

Beck, A.T., \& Emery, G. (1985). Anxiety disorders and phobias: A cognitive perspective. New York: Basic Books

Bennion, L.D., \& Adams, G.R. (1986). A revision of the extended version of the Objective Measure of Ego Identity Status: An identity instrument for use with late adolescents. Journal of Adolescent Research, 1, 183-198.

Bradley, G., \& Matsukis, P. (2000). Identity status and youth recklessness. Youth Studies Australia, 19(4), 48-54

Cox, B.J., Ross, L., Swinson, R.P., \& Direnfeld, D.M. (1998). A comparison of social phobia outcome measures in cognitive-behavioral group therapy. Behavior Modification, 22, 285-297.

Danielsen, L.M., Lorem, A.E., \& Kroger, J. (2000). The impact of social context on the identityformation process of Norwegian late adolescents. Youth and Society, 31(3), 332-363.

Fresco, D.M., Coles, M.E., Heimberg, R.G., Liebowitz, M.R., Hami, S., Stein, M.B., et al. (2001). The Liebowitz Social Anxiety Scale: A comparison of the psychometric properties of the selfreport and clinician-administered formats. Psychological Medicine, 31, 1025-1035.

Griffiths, M. (1997). Friendship and social development in children and adolescence: The impact of electronic technology. Education and Child Psychology, 14(3), 25-37.

Kashdan, T.B., \& Herbert, J.D. (2001). Social anxiety disorder in childhood and adolescence: Current status and future directions. Clinical Child and Family Psychology Review, 4(1), 37-61.

Kraut, R., Patterson, M., Lundmark, V., Kiesler, S., Mukophadhyay, T., \& Scherlis, W. (1998). Internet paradox: A social technology that reduces social involvement and psychological well-being? American Psychologist, 53, 1017-1031.

Kroger, J. (2000). Ego identity status research in the new millennium. International Journal of Behavioral Development, 24(2), 145-148.

La Greca, A.M., \& Lopez, N. (1998). Social anxiety among adolescents: Linkages with peer relations and friendships. Journal of Abnormal Child Psychology, 26, 83-94.

La Rose, R., Eastin, M.S., \& Gregg, J. (2001). Reformulating the Internet paradox: Social cognitive explanations of Internet use and depression. Journal of Online Behavior, 1(2). Retrieved August 25, 2002, from http://www.behavior.net/JOB/vlnl/paradox.html

Liebowitz, M.R. (1987). Social phobia. Modern Problems in Pharmacopsychiatry 22,141-173.

McKenna, K.Y.A., \& Bargh, J.A. (1998). Coming out in the age of the Internet: Identity 'demarginalization' through virtual group participation. Journal of Personality and Social Psychology, 75, 681-694.

McKenna, K.Y.A., \& Bargh, J.A. (1999). Causes and consequences of social interaction on the Internet: A conceptual framework. Media Psychology, 1, 249-270.

McKenna, K.Y.A., \& Bargh, J.A. (2000). Plan 9 from cyberspace: The implications of the Internet for personality and social psychology. Personality and Social Psychology Review, 4, 57-75.

Moody, E.J. (2001). Internet use and its relationship to loneliness. Cyber Psychology and Behavior, 4, 393-401. 
Parks, M.R., \& Floyd, K. (1995). Making friends in cyberspace. Journal of Communication, 46, 80-97.

Schwartz, S.J., Mullis, R.L., Dunham, R.M., \& Waterman, A.S. (2000). Ego identity status, identity style, and personal expressiveness: An empirical investigation of three convergent constructs. Journal of Adolescent Research, 15, 504-522.

Shashaani, L. (1997). Gender differences in computer attitudes and the use among college students. Journal of Educational Computing Research, 16, 37-51.

Short, J., Williams, E., \& Christie, B. (1976). The social psychology of telecommunications. London: Wiley.

Stein, M.B. (2001). Unmasking social anxiety disorder. Journal of Psychiatry and Neuroscience, 26, $185-190$.

Stein, M.B., Fuetsch, M., Muller, N., Hofler, M, Lieb, R., \& Wittchen, H.U. (2001). Social anxiety disorder and the risk of depression: A prospective community study of adolescents and young adults. Archives of General Psychiatry, 58, 251-263.

Utz, S. (2000). Social information processing in MUDs: The development of friendships in virtual worlds. Journal of Online Behavior, 1(1). Retrieved August 25, 2002, from http://www./JOB/ v1n1/utz.html

Wallace, P. (1999). The psychology of the Internet. Cambridge, UK: Cambridge University Press.

Walsh, J. (2002). Shyness and social phobia. Health and Social Work, 27, 137-145.

Walther, J.B. (1997). Group and interpersonal effects of international computer-mediated collaboration. Human Communication Research, 23, 342-369.

Waterman, A.S. (1982). Identity development from adolescence to adulthood: An extension of theory and a review of research. Developmental Psychology, 18, 341-358.

Wolfradt, U., \& Doll, J. (2001). Motives of adolescents to use the Internet as a function of personality traits, personal and social factors. Journal of Educational Computing Research, 24, 13-27.

Young, K.S. (1996). Psychology of computer use: XL. Addictive use of the Internet: A case that breaks the stereotype. Psychological Reports, 79, 899-902. 